\title{
Transatlantica
}

Revue d'études américaines. American Studies Journal

Hors-série | 2021

Numéro anniversaire : 20 ans de la revue

\section{Introduction. 20 ans ? 20 ans !}

\section{Guillaume Marche et Cécile Roudeau}

\section{(2) OpenEdition}

\section{Journals}

Édition électronique

URL : https://journals.openedition.org/transatlantica/17488

DOI : 10.4000/transatlantica. 17488

ISSN : $1765-2766$

\section{Éditeur}

Association française d'Etudes Américaines (AFEA)

\section{Référence électronique}

Guillaume Marche et Cécile Roudeau, «Introduction. 20 ans ? 20 ans !», Transatlantica [En ligne], Horssérie | 2021, mis en ligne le 01 octobre 2021, consulté le 11 octobre 2021. URL : http://

journals.openedition.org/transatlantica/17488; DOI : https://doi.org/10.4000/transatlantica.17488

Ce document a été généré automatiquement le 11 octobre 2021.

\section{(c) (i) (9)}

Transatlantica - Revue d'études américaines est mise à disposition selon les termes de la licence Creative Commons Attribution - Pas d'Utilisation Commerciale - Pas de Modification 4.0 International. 


\title{
Introduction. 20 ans ? 20 ans !
}

\author{
Guillaume Marche et Cécile Roudeau
}

1 Est-on vraiment sérieux quand on a vingt ans? Rédactrice et rédacteur en chef de Transatlantica en 2021, il nous incombe la lourde, mais plaisante, responsabilité de célébrer cet important anniversaire de la revue dont on nous a confié les rênes il y a quelques années. Pour l'avoir vue prendre son essor à mesure que nous-mêmes entrions dans la profession d'enseignant•e-chercheur•se en études américaines, nous répondons d'abord par un immense «oui»! De revue "junior» et expérimentale publiée sous les auspices de l'Association Française d'Etudes Américaines, Transatlantica s'est vite imposée, aux côtés de sa grande sœur imprimée, la Revue Française d'Études Américaines, de 25 ans son aînée, comme l'un des repères majeurs du champ des études américaines en France. Et c'est donc pour nous une grande fierté de saluer le rôle qu'elle joue auprès de la communauté des chercheurs et chercheuses américanistes, en France et au-delà. Car s'il est un atout de la publication en ligne dont Transatlantica a su se saisir, c'est bien de se rire des frontières, comme des contraintes que sont le nombre de pages, de mots, de signes. Son lectorat parcourt ses pages depuis la France, les ÉtatsUnis, mais aussi l'Allemagne ou l'Inde. L'origine de ses contributions s'est également diversifiée au fil du temps, avec de plus en plus d'auteurs et d'autrices établi•es en Amérique du Nord et dans d'autres pays d'Europe, à mesure que sa fréquentation a crû de manière importante - de presque 260000 visites depuis 140000 ordinateurs différents en 2015 à plus de 370000 depuis quelque 292000 ordinateurs différents en 2020, 3 à $4 \%$ des visites durant plus d'une demi-heure. À mesure aussi que l'anglais y a pris une part aujourd'hui prépondérante - sans être toutefois exclusive, car le comité de rédaction tient à ce que la revue reste aussi un vecteur francophone de la recherche en études américaines.

2 S'entretenant pour la préparation de ce numéro avec Olivier Brossard, Jean Kempf, cofondateur de la revue, avec François Brunet et quelques autres, nous a rappelé qu'elle est née du souhait de faire paraître un genre de super bulletin ou un annuaire augmenté, de recenser, en somme, ce qui se faisait en études américaines en France. "L'idée, au départ, nous dit Jean Kempf, ce n'était pas une idée révolutionnaire, c'était un peu de porter la bibliographie annuelle, et aussi les annonces de colloques en temps réel ou presque, c'était de faire une newsletter qui pourrait être envoyée sans que cela 
ait des coûts postaux considérables. » Efficacité, réactivité et économie étaient donc les maitres mots de ce projet. Avec un numéro par an pendant 4 ans, puis deux par an depuis 2006, Transatlantica s'enorgueillit à ce jour d'un sommaire complet de 34 numéros, construits autour d'un et souvent deux dossiers thématiques, et d'un nombre de rubriques qui a évolué au fil du temps. Elles sont 6 à ce jour. Efficacité, réactivité et économie ont parfois été sacrifiées au profit du sérieux éditorial, quand certains numéros sont parus jusqu'à deux ans après la date qu'ils portent. Et ce, pour garantir la qualité scientifique qu'offrent non seulement les deux évaluations en double aveugle, auxquelles sont soumis les articles de recherche, mais aussi les riches échanges avec les auteurs et autrices, et avec les coordinateurs et coordinatrices, au fil desquels les articles évoluent, s'enrichissent et prennent parfois une certaine ampleur, voire un certain embonpoint...

3 Alors, vénérable organe des études américaines en France, déjà, Transatlantica ? Certainement, mais pas seulement. Jean Kempf nous rappelle que c'est la volonté d'expérimenter, de prendre des risques - celui de ne pas plaire à tout le monde, par exemple - qui a présidé à sa naissance. Transatlantica, c'était un peu l'enfant terrible. Si la revue n'a pas introduit les études visuelles ou musicales dans le champ des études américaines en France, elle a assurément accompagné leur essor avec ses rubriques "Trans'arts» et "La boite à musique ». Les américanistes ont-ils et elles besoin d'ouverture vers les approches innovantes qui renouvellent notre champ ? Veulent-ils ou elles plus de réflexivité sur les travaux fondateurs, les chercheuses et chercheurs qui ont donné ses titres de noblesse aux études américanistes, ou sur les œuvres, autrices et auteurs qui lui donnent sa raison d'être? «Reconnaissances » et "Perspectives » sont là qui veillent, et donnent matière à penser, tandis que "Recensions " et "Actualité de la recherche", en dignes héritières du projet initial d'avant même l'invention d'Internet, nous orientent dans le riche foisonnement protéiforme que sont les études américaines.

4 Ni réplique virtuelle d'une revue imprimée ni simple site web de promotion scientifique, la revue a dû apprendre à concilier la souplesse du format électronique et le besoin de pérennité, de reconnaissance, de légitimation qu'accrédite la publication écrite en lettres, et sciences humaines et sociales. Mais le bricolage - intellectuel, technologique, institutionnel - dont Transatlantica est le fruit s'accommode fort bien de la créativité des américanistes. Le congrès 2016 de l'AFEA n'a-t-il pas été intitulé "Chantiers d'Amérique », tandis que celui de 2019 nous invitait à l' "Indiscipline »? Revue sérieuse sans être une revue classique, en prise avec l'actualité - littéraire, artistique, sociale, politique... - sans en être ni l'esclave ni un simple reflet, Transatlantica se donne, et veut donner à ses lecteurs et lectrices, comme à ses auteurs et autrices, le temps de la réflexion. Tout en s'autorisant à publier vite. Certains textes ne peuvent attendre, car les jeunes chercheuses et chercheurs sont de plus en plus soumis•es à la nécessité de faire connaitre leurs travaux. Mais, Transatlantica se hâte lentement, aussi parce qu'il faut laisser du temps à ceux et celles qui lisent, relisent, conseillent, à ceux et celles qui apparient patiemment un texte et un lecteur, une lectrice.

Car oui, disons-le, Transatlantica aime susciter les rencontres, fortuites ou non, et les longs compagnonnages qui parfois en découlent, les rencontres anonymes, d'un rivage à l'autre de l'Atlantique, d'une génération à l'autre, aussi. Quel•le jeune chercheur•se n'a pas bénéficié des conseils avisés d'un•e ainé•e? Et quel•le chercheur•se 
chevronné•e n'a pas été piqué au vif par les critiques, constructives, certes, mais parfois sans ménagement, que lui prodiguait sous couvert d'anonymat un•e expert•e bien moins installé•e dans la profession, mais dont la familiarité avec des approches plus récentes lui donnait un regard acéré et percutant? Transatlantica est fière de mettre le pied à l'étrier à ces jeunes américanistes qui lui soumettent des articles, se voient confier des expertises, coordonnent des dossiers ou intègrent son comité de rédaction.

Mais place, donc, au numéro. Ce numéro spécial est le fruit d'une volonté collective, celle du comité de rédaction, dont la rédactrice et le rédacteur en chef ne sont ici que les porte-paroles, de faire quelque chose de différent, de briser un peu les cadres. Tout en restant sérieux... on a vingt ans, tout de même! Nous y avons invité nos prédécesseur•es à partager librement leurs souvenirs, mais nous avons aussi souhaité recueillir le point de vue de nos homologues d'autres revues d'études américaines sur le sens, les défis et l'avenir de la publication universitaire dans notre domaine. Cet anniversaire est également l'occasion de donner la parole à quelques membres de notre comité scientifique, établi•es aux États-Unis, mais aussi en Allemagne ou en Australie, qui nous ont accordé un entretien pour échanger sur leur travail et, plus généralement, sur la portée scientifique, sociétale, voire politique de la recherche dans les divers domaines qui participent aux études américaines. Regards rétrospectifs et souvenirs partagés prennent aussi la forme d'images, juste légendées, que nous ont livrées une trentaine d'entre vous, lecteurs et lectrices, contributeurs et contributrices. Ce numéro, enfin, ne ressemblerait pas à notre revue s'il n'accordait une place de choix aux plus jeunes membres de notre communauté scientifique : il comporte donc un état des lieux réflexif de jeunes chercheurs et chercheuses sur le rôle des organismes et des institutions qui structurent les études américaines en France et en Europe.

7 Alors, dans vingt ans... ? Toni Morrison disait dans l'une de ses harangues : « If there's a book that you want to read, but it hasn't been written yet, then you must write it.» Transatlantica est un peu ce livre, ouvert, collaboratif. De nouveaux chantiers s'ouvrent déjà, de nouveaux défis, que Transatlantica est prête à relever - de sa position liminale, bilingue, interdisciplinaire, intermédiale, transatlantique. Ce pas de côté, nous le revendiquons, comme nous revendiquons une pensée critique qui continuera, nous l'espérons, de s'exercer à travers de nouvelles formes, sur de nouveaux périmètres. À l'heure où certaines frontières, même numériques, se ferment, Transatlantica veut être plus que jamais passe-muraille, engagée dans la pensée et dans le monde, un brin téméraire, voire provocatrice, "one of the roughs». Et si on lui souhaitait d'avoir toujours vingt ans?

\section{AUTEURS}

\section{GUILLAUME MARCHE}

Université Paris-Est Créteil, IMAGER 


\section{CÉCILE ROUDEAU}

Université de Paris, LARCA 